\title{
Mutant HIV-1 Protease Complexed with Tetrapeptide Inhibitor. Preliminary Report
}

\author{
T. SkÁlovía ${ }^{a, *}$, J. HašeK ${ }^{a}$, J. DohnÁlek ${ }^{a}$, H. Petroková ${ }^{a}$ \\ AND E. BUChTELOVÁ ${ }^{b}$ \\ ${ }^{a}$ Institute of Macromolecular Chemistry AS CR \\ Heyrovskeho nam. 2, 16206 Prague 6, Czech Republic \\ ${ }^{b}$ Faculty of Science, Charles University, Prague, Czech Republic
}

\begin{abstract}
The paper presents preliminary description of structure determination of a mutant form of HIV-1 protease in a complex with a new pseudotetrapeptide inhibitor that retains its high affinity to this mutant enzyme (A71V, V82T, $\mathrm{I} 84 \mathrm{~V})$. A highly efficient microdiffractometer installed for our experiment at the beamline ID14-1 enabled us to collect satisfactory data with crystal dimensions of about 40 micrometers.
\end{abstract}

PACS numbers: 87.15. By

\section{Introduction}

Inhibition of HIV protease is (besides inhibition of reverse transcriptase) one of two ways that are used to break the life cycle of the human immunodeficiency virus to stop progress of the AIDS disease. Six drugs against AIDS based on inhibition of HIV protease have already been clinically approved, however, a rapid development of mutant forms of the protease hinders their long term efficiency. The group of Konvalinka found, by combinatorial chemistry, a series of inhibitors showing good inhibition properties ( $K_{i}$ values in a nanomolar or subnanomolar range) for native and also for tested mutant forms isolated from patients treated by officially approved drugs [1-3].

*corresponding author; e-mail: skalova@imc.cas.cz 


\section{Experimental}

This contribution presents structure refinement of mutant HIV-1 protease (mutations $\mathrm{A} 71 \mathrm{~V}, \mathrm{~V} 82 \mathrm{~T}, \mathrm{I} 84 \mathrm{~V}$ ) in complex with the pseudopeptide inhibitor of ethylamine type Boc-Phe- $\psi\left[\mathrm{CH}_{2} \mathrm{CH}_{2} \mathrm{NH}\right]-\mathrm{Phe}-\mathrm{Glu}-\mathrm{Phe}-\mathrm{NH}_{2}$ (Fig. 1). The inhibitor can be derived from a potential substrate by replacing the cleavable peptide bond by isosteric ethylamine group $\mathrm{CH}_{2} \mathrm{CH}_{2} \mathrm{NH}$. The inhibitor (denoted as $\mathrm{OE}-$ no hydroxy group and Glu in position $P_{2}^{\prime}$ ) was prepared by Hradilek [2]. The mutant protease, developed in patients under selection pressure of Indinavir [1], was prepared and purified in the laboratory of Konvalinka. In vitro tests of inhibition properties were published in $[1,2]$.

TABLE

Results of data processing.

\begin{tabular}{l|c}
\hline \hline Space group & $P 6_{1}$ \\
Unit cell: $a, b, c[\AA]$ & $62.68,62.68,83.31$ \\
\multicolumn{1}{c|}{$\alpha, \beta, \gamma\left[^{\circ}\right]$} & $90,90,120$ \\
Resolution & $33-2.2 \AA$ \\
No. of measurements & 61979 \\
No. of unique reflections & 9490 \\
Completeness & $99.9 \%$ \\
Mosaicity & $0.19^{\circ}$ \\
$R_{\text {merge }}$ & $7.9 \%$
\end{tabular}

The complex of the mutant protease with the inhibitor OE was crystallized under similar conditions as in [4]. The X-ray diffraction data of the crystal were collected at the synchrotron facility ESRF in Grenoble on the beamline ID14-1 at a temperature of $100 \mathrm{~K}$ using a wavelength $\lambda=0.934 \AA$. The local microdiffractometer with the primary beam diameter of $30 \mu \mathrm{m}$ enabled measurements on three different sites of the needle-like sample $(40 \times 50 \times 300 \mu \mathrm{m})$. In total, 73 images were recorded with a $165 \mathrm{~mm}$ MAR CCD detector with oscillation angle $1^{\circ}$, exposition time $30 \mathrm{~s}$ and crystal to detector distance $150 \mathrm{~mm}$.

Data were processed using software packages HKL [5] and CCP4 [6]. The results are presented in the Table. The space group of the crystal is $P 6_{1}$ with one dimer of protease in the asymmetric unit. The structural model is being refined using the software package CNS [7] and program $\mathrm{O}[8]$.

\section{Results}

It is known that HIV-1 protease is a dimer composed of two chemically identical and conformationally similar chains of 99 amino acid residues with the active site (responsible for cleavage function of the protease) at aspartates Asp A25 and Asp B125. 


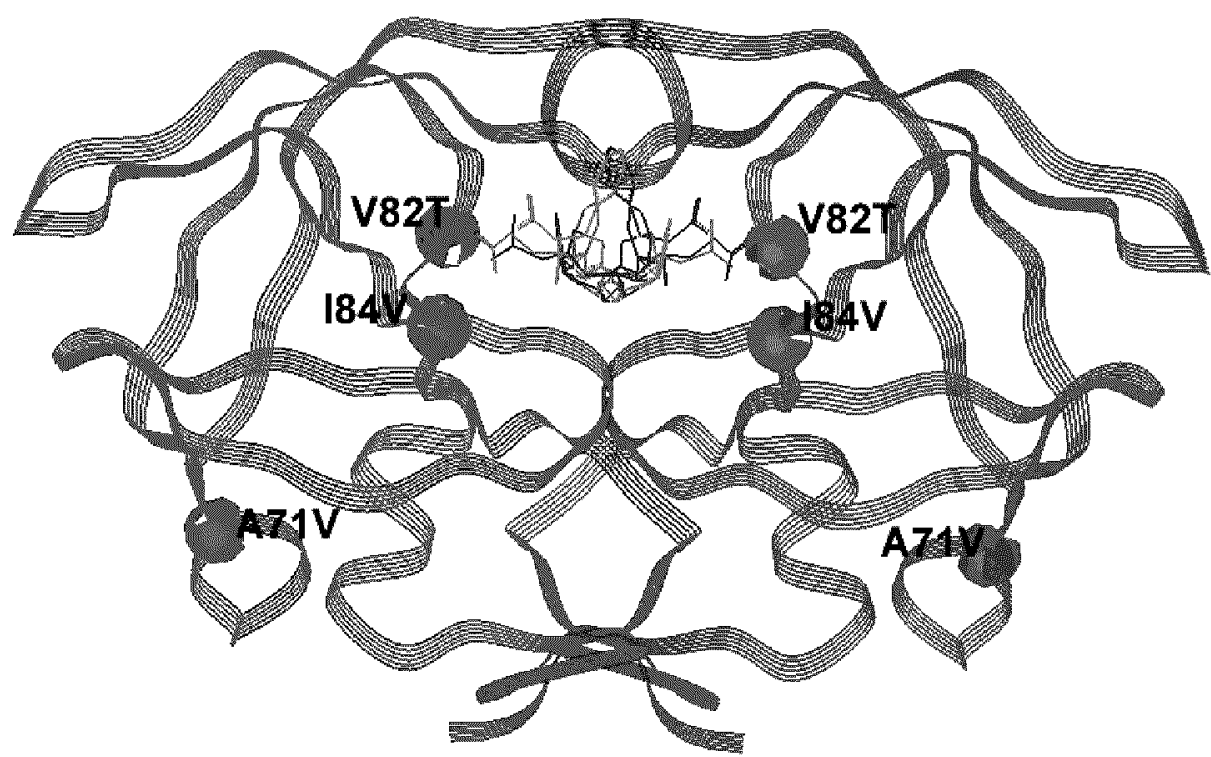

Fig. 1. HIV-1 protease with denoted positions of mutant residues. Inhibitor OE is bound in two opposite orientations in the active site.

The relatively rigid protease dimer tends to preserve its approximate twofold symmetry. However, there are some reasons for perturbation of this twofold symmetry in our case. First, the spiral stacking of molecules in space group $P 6_{1}$ causes different intermolecular contacts and thus different conformation of some side chains on opposite sides of dimer. And secondly, the inhibitor alone has no symmetry at all and thus, we can expect some variations in the side chains mediating the interactions of protease with the inhibitor. In our structure (contrary to the native structure with the same inhibitor $\mathrm{OE}$ ) the mutated residues 82,84 , situated in the binding tunnel, have two alternative conformations (Fig. 2).

Further, the fact that the inhibitor is almost completely buried under the protease flaps and has negligible contacts to neighbor complexes forming the crystal, leads frequently to 1:1 disorder in the inhibitor orientation in the binding tunnel of protease. Our inhibitor was clearly localized in the difference map in two opposite orientations. Occupation factors were refined close to 0.5 for both orientations.

Free refinement of all parameters required for this model at $2.2 \AA$ resolution would be problematic. To avoid overfitting, the non-crystallographic restraints were imposed on the whole protease dimer in the starting model. These were later removed for all residues where the difference maps confirmed an existence of different conformations on both sides of the protease. Not disordered residues fitting well the electron density were refined further with the non-crystallographic twofold symmetry restraints on positions with the effective energy of 


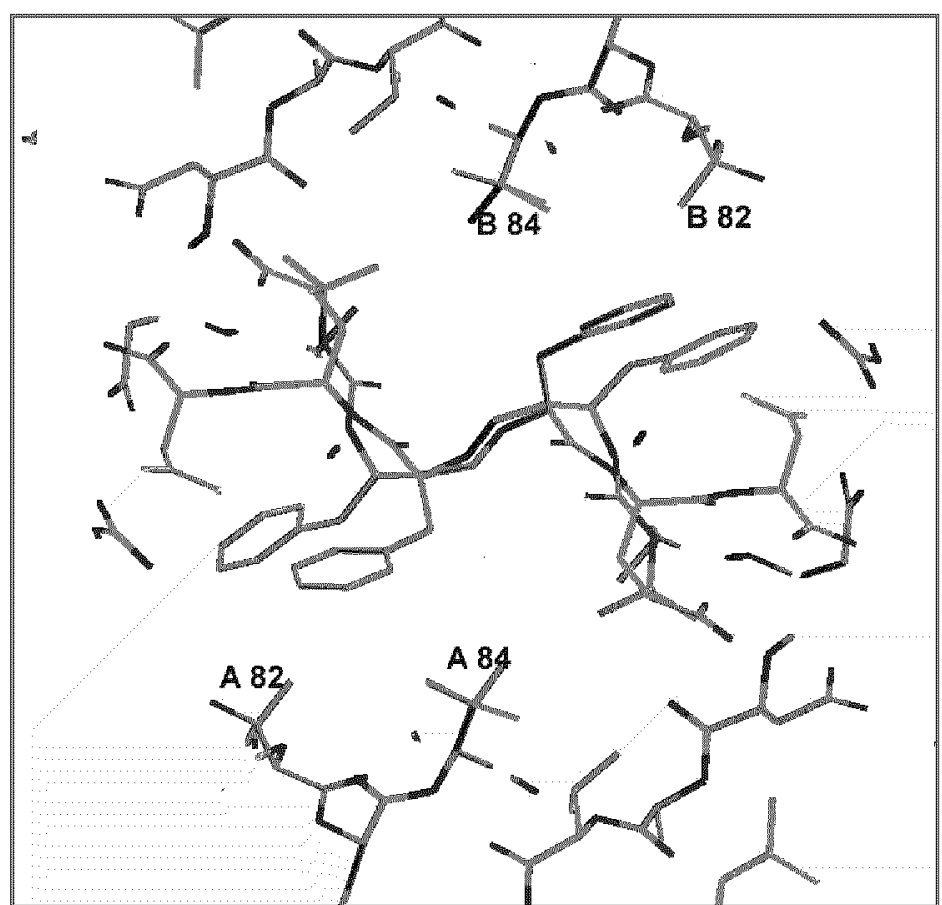

Fig. 2. The active site with bound inhibitor in a view from flaps of the protease. Mutant residues, denoted in both chains by sequence numbers of residues, are in alternative conformations.

$200 \mathrm{kCal} \mathrm{mol}^{-1} \AA^{-2}$.

The present model includes the protease, the inhibitor and 55 water molecules giving $R$-factors: $R=22.2$ and $R_{\text {free }}=25.9$.

\section{Conclusion}

The molecular mechanics calculations propose, and the X-ray data support, the existence of conformational disorder in some parts of molecular structure even in the inhibitor binding tunnel. The final refinement, the study of interactions and the discussion of mobility of some structure segments will be published later.

\section{Acknowledgment}

Authors thank J. Konvalinka, M. Hradilek, and J. Weber from IOCB AS CR for providing the mutated protease and the inhibitor OE for crystallization experiments. The research was supported by the project A4050811/1998 of the Grant Agency AS CR and projects of GA CR 203/98/K023, 203/00/D117, and 204/00/P091. 


\section{References}

[1] J. Weber, Ph.D. Thesis, Faculty of Sciences, Charles University, Prague 2001.

[2] J. Konvalinka, J. Litera, J. Weber, J. Vondrášek, M. Hradílek, M. Souček, I.Pichová, P. Majer, P. Štrop, J. Sedláček, A.M. Heuser, H. Kottler, H.G. Krausslich, Eur. J. Biochem. 250, 559 (1997).

[3] T. Skálová, J. Hašek, J. Dohnálek, H. Petroková, E. Buchtelová, Mater. Struct. 8, 29 (2001).

[4] J. Dohnálek, J. Hašek, J. Dušková, H. Petroková, M. Hradílek, M. Souček, J. Konvalinka, J. Brynda, J. Sedláček, J. Fábry, Acta Crystallogr. D 57, 472 (2001).

[5] Z. Otwinowski, in: Proc. CCP4 Study Weekend: Data Collection and Processing, Eds. L. Sawyer, N. Isaacs, S. Bailey, SERC Daresbury Laboratory, Daresbury 1993, p. 56.

[6] The CCP4 Suite: Programs for Protein Crystallography, Acta Crystallogr. D 50, 760 (1994).

[7] A.T. Brunger, P.D. Adams, G.M. Clore, W.L. Delano, P. Gros, R.W. Grosse-Kunstleve, J.-S. Jiang, J. Kuszewski, N. Nilges, N.S. Pannu, R.J. Read, L.M. Rice, T. Simonson, G.L. Warren, Acta Crystallogr. D 54, 905 (1998).

[8] T.A. Jones, M. Kjeldgaard, Manual for O version 5.9, Department of Molecular Biology, BMC, Uppsala University, Sweden; Department of Chemistry, Aarhus University, Denmark 1993. 